\title{
Capítulo
}

4

\section{Procedimentos adotados na coleta de sinais mioelétri- cos para construção de próteses da mão}

\author{
Alberto Monteiro Peixoto, Guilherme de Oliveira Monteiro Peixoto, Roberto \\ Luiz Souza Montteiro, Tereza Kelly Gomes Carneiro
}

\begin{abstract}
This chapter provides information on the precautions and recommendations that need to be taken in collecting the myoelectric signal for use in myoelectric prostheses with multiple movements. For a better understanding of the process, an approach is made about the EMG signal, its treatment, movement identification techniques and the instructions for the acquisition process. A prototype acquisition equipment is also being presented here as an example.
\end{abstract}

\section{Resumo}

Este capítulo traz informações sobre os cuidados e recomendações que precisam ser tomados na coleta do sinal mioelétrico para uso em próteses mioelétricas com vários movimentos. Para melhor compreensão do processo, é feita uma abordagem sobre o sinal EMG, seu tratamento, técnicas de identificação dos movimentos e as instruções para o processo de aquisição. Aqui também está sendo apresentado um protótipo de equipamento de aquisição como exemplo.

\subsection{Informações Gerais}

Este capítulo tem o objetivo de apresentar o processo de aquisição dos sinais mioelétricos, abordando as condutas que devem ser adotadas para se conseguir maximizar a identificação do número de movimentos realizados pelo membro superior.

A abordagem está organizada de forma a proporcionar, não só o conhecimento das principais condutas que deverão ser adotadas para a identificação dos movimentos, como também a compreensão do processo de captura do sinal. Desta forma, o capítulo está estruturado em cinco subtítulos: Identificação da atividade muscular; Características do sinal mioelétrico; Tratamento dos sinais mioelétricos; Técnicas de identificação dos movimentos; e Instruções para o processo de aquisição. 


\subsection{Identificação da Atividade Muscular}

Uma das condições importante para compreensão e melhor aquisição dos sinais mioelétricos é o entendimento de como os vários músculos contribuem para realização de um determinado movimento, pois o sinal eletromiográfico (EMG), é formado por um conjunto de sinais provenientes de músculos que estão atuando diretamente no movimento investigado, como também de músculos que estão atuando indiretamente.

Portanto, abordaremos a seguir como os músculos contribuem com os movimentos e quais os principais mecanismos que atualmente podem ser utilizados para detectá-los.

\subsubsection{Atividade muscular e sua relação com os movimentos}

Para entendermos a participação dos músculos em determinados movimentos, utilizaremos a seguir a classificação apresentada por (LIPPERT, 2013) que distingue quatro tipos de músculos:

Agonistas: Este termo é aplicado aos músculos que são os principais responsáveis por um determinado movimento do membro. Se considerarmos, por exemplo, flexão do antebraço sobre o braço, o bíceps braquial seria o agonista.

Antagonistas: Este termo é aplicado aos músculos que agem realizando movimento oposto aos agonistas. Durante a ação de um agonista, os antagonistas geralmente atuam passivamente sem se contrair nem oferecer resistência. Se considerarmos o exemplo da flexão do antebraço sobre o braço, o tríceps braquial seria o antagonista.

Neutralizadores: Este termo é aplicado aos músculos que atuam junto aos agonistas para permitir que um determinado movimento seja executado de forma adequada. Se considerarmos o exemplo da flexão do antebraço sobre o braço, percebemos que o bíceps braquial (agonista) faz a flexão do antebraço sobre o braço, mas produz também o movimento de supinação. Para evitar este segundo movimento, outro músculo atua com o objetivo de neutralizá-lo. Neste caso, o músculo pronador redondo.

Estabilizadores: Este termo é aplicado aos músculos que atuam estabilizando as estruturas do corpo para que determinado movimento seja realizado. Considerando o exemplo da flexão do antebraço sobre o braço, para que este movimento seja feito para realizando um levantamento de peso sem que a articulação do braço sofra deslocamento, o músculo deltoide precisará atuar estabilizando a articulação do ombro (glenoumeral). Portanto, neste caso o deltoide é um musculo estabilizador.

Sendo assim, o sinal mioelétrico capturado na superfície da pele é formado por um complexo conjunto de sinais originados em vários tipos de músculos. Alterações posturais, mudanças na angulação do movimento ou a presença de movimentos paralelos são fatores que podem alterar o padrão do sinal mioelétrico.

Esta informação nos permite entender porque os movimentos precisam ser treinados junto aos processos de aquisição do sinal EMG. Quanto mais treinos, mais bem definido o padrão do sinal capturado e maior a probabilidade de diferenciar um número maior de movimentos. 


\subsubsection{Mecanismos utilizados para detecção das atividades musculares}

Embora o sinal mioelétrico seja a informação mais completa sobre a atividade muscular, existem vários métodos que podem ser utilizados para detectar essa atividade. Abordaremos aqui quatro destes métodos: eletromiografia; panda ring resonator; optomiografia e mecanomiografia.

Eletromiografia: Esta é uma técnica utilizada para captura dos sinais elétricos produzidos pelos músculos. A eletromiografia (EMG) pode ser realizada de duas formas: utilizando eletrodos de agulha (IEMG) ou utilizando eletrodos de superfície (SEMG). A forma mais utilizada desta técnica para o uso em próteses é a SEMG, visto que tem a vantagem de ser não invasiva (HARGROVE; ENGLEHART; HUDGINS, 2007).

A SEMG utiliza eletrodos de superfície para fazer a captura dos sinais EMG e a escolha do seu formato, assim como a composição vão depender do objetivo da coleta. A Figura 4.1 apresenta alguns formatos destes eletrodos. Em (a) é apresentado tipo bipolar com barras de $\mathrm{Ag} / \mathrm{AgCl}$, sendo um formato utilizado de forma fixa em algumas próteses; em (b) é apresentado um tipo também bipolar com pinos mais afastados; em (c) está apresentado o tipo descartável monopolar, bastante utilizado por ser de baixo preço e de fácil uso, no entanto, o cabo é conectado ao eletrodo através de um conector, o que acaba produzindo ruídos causados por artefatos de movimento; e em (d) temos também um modelo monopolar, sendo diferente do anterior por ter o cabo fixado no eletrodo, evitando os artefatos de movimento.

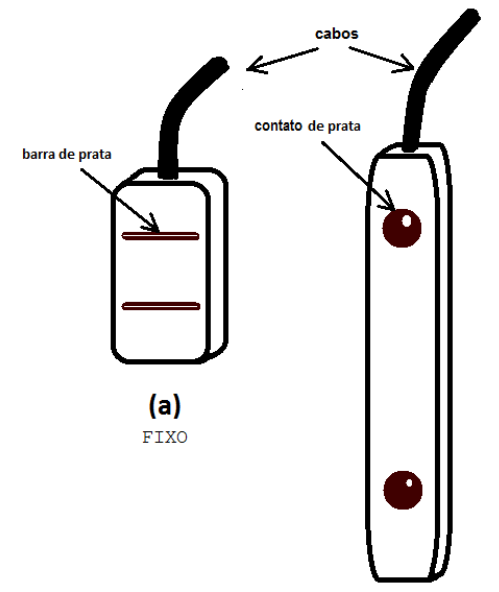

(b)

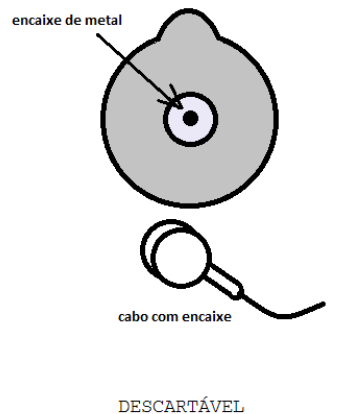

(c)

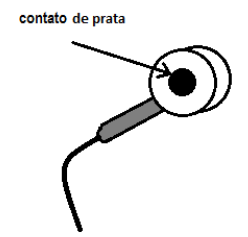

(d)

Figure 4.1. Visão geral de alguns tipos de eletrodos utilizados na SEMG.

Estes eletrodos são componentes muito importantes na aquisição dos sinais, visto que são eles que permitem o contato entre a pele e o sistema de captura do sinal.

O contato eletrodo-pele pode ser um gargalo na obtenção do sinal mioelétrico devido à impedância apresentada nesta conexão. $\mathrm{O}$ aumento da impedância pode ser produzido tanto por uso de um eletrodo inadequado, quanto por fatores biológicos inerentes à pele.

Os eletrodos mais utilizados para captura dos sinais mioelétricos com o objetivo de 
uso em próteses, são os que utilizam os contatos de $\mathrm{Ag} / \mathrm{AgCl}$, tanto para os eletrodos fixos quanto para os descartáveis. No caso dos descartáveis, são utilizados os mesmos eletrodos indicados para a aquisição dos sinais cardíacos (ECG). Estes eletrodos utilizam, além dos contatos de $\mathrm{Ag} / \mathrm{AgCl}$, gel eletrolítico. Outros procedimentos também são adotados para baixar a impedância da interface eletrodo-pele, que são a retirada de pelos da pele, e a limpeza da superfície para retirada de cremes, suor e tecidos mortos.

O uso da eletromiografia é bastante ampla, perpassando por diagnóstico de doenças; estudo dos movimentos musculares; construções de equipamentos de biofeedback; e para a construção de próteses mioelétricas.

Panda Ring Resonator: Trata-se de um sensor óptico que tem potencial para ser empregado na detecção de movimentos musculares graças a variação da fase e comprimento de onda ocasionados pela perturbação no caminho óptico. Ele é constituído por três ressonadores de micro-anel estruturados como unidade de referência (RL), unidade de detecção (RR) e o terceiro é usado para formar sinais de interferência.

Com esse sistema óptico de detecção muscular somos capazes de identificar facilmente quando ocorre ou não uma perturbação no sistema. Quando não ocorre perturbação, a simetria entre os picos do sinal de referência e o sinal de detecção não mudam. Caso ocorra perturbação, o sinal da unidade de detecção (RR) que foi perturbada pela contração muscular, possuirá um comprimento de onda levemente diferente da unidade de referência.

A relação entre a mudança no comprimento de onda e a perturbação no comprimento do caminho óptico causada pela contração muscular é dada de forma proporcional. Como mostra a Equação (1).

$$
\frac{\Delta \lambda m}{\lambda m}=\frac{\Delta n}{n}+\frac{\Delta L}{L}
$$

Em que L é o comprimento do caminho óptico, ou seja, a circunferência do ring resonator, $n$ é o índice de refração. Já a relação entre a força aplicada e a variação do comprimento do caminho óptico depende também do módulo de Young e da área da secção transversal. Como mostra a Equação (2).

$$
F=\left(\frac{Y_{0} A_{0}}{L_{0}}\right) \cdot \Delta L
$$

Em que $\mathrm{F}$ é a força aplicada, $Y_{0}$ é o módulo de Young, $A_{0}$ é a área inicial da secção transversal, $L_{0}$ é o comprimento inicial e $\Delta L$ é a variação no comprimento óptico. Em [1] e [2] identificamos uma relação linear entre a força aplicada e a variação do comprimento de onda. Essa relação linear torna viável o uso do sistema óptico de detecção muscular (YOTHAPAKDEE; P.YUPAPIN; TAMEE, 2016).

Assim como em outros sensores de deformação, o ring resonator se baseia também no efeito óptico de tensão (strain-optical effect) que consiste na variação do índice de refração induzido por qualquer deformação aplicada à fibra óptica (TAMEE et al., 2013).

Optomiografia: A optomiografia é uma técnica que pode ser utilizada para iden- 
tificar a atividade muscular. O sensor eletro-óptico para detecção de contração muscular apresentado por Chianura e Giardini (2010) se embasa na observação de que as células musculares são acomodadas como fibras alongadas, alinhadas ao longo do eixo principal do músculo. No tecido muscular, espera-se que a luz infravermelha, seja retroespalhada anisotropicamente conforme o músculo se contrai.

O sensor é colocado diretamente na pele, sobre o músculo. Um LED localizado no centro do sensor emite luz através da pele e dois fotodiodos não adjacentes a coletam na direção das fibras, enquanto outros dois fotodiodos coletam a luz espalhada perpendicularmente a essa direção.

Este tipo de sensor óptico parece promissor como uma alternativa ao EMG de superfície, permitindo uma detecção não invasiva do sinal de contração muscular, com vantagens na não captação de ruído eletromagnético e com a capacidade de distinguir entre contrações isométricas e isotônicas.

\section{Mecanomiografia:}

Refere-se a uma técnica não-invasiva utilizada para identificar contração muscular. Distintos instrumentos podem ser utilizados como: ondas sonoras e vibrações. Keidel e Keidel (1989) empregou o termo vibromiografia para os detectores de vibração, e Orizio, Perini e Veicsteinas (1989) empregou o termo sonomiografia para os detectores sonores. Este mesmo autor empregou o termo Mecanomiografia em 1993 que passou a englobar todas as técnicas não-invasivas de detecção da atividade muscular através de vibrações e sons medidos por transdutores.

Outro exemplo de instrumento pode ser visto no trabalho de Krueger et al. (2014) que utiliza acelerômetros com três eixos para medir as vibrações de deslocamento geral da fibra muscular nas três direções ortogonais (X, Y e Z). Já no trabalho de Watakabe et al. (2001) é mostrado a diferença entre sinais mecanomiograma (MMG) capturados utilizando um microfone e um acelerômetro, evidenciando que as características do sinal MMG dependem do tipo do sensor utilizado.

Por fim, embora várias técnicas para detecção da atividade motora tenha surgido nos últimos anos, a técnica mais utilizada e que tem se mostrado a mais adequada para identificação dos movimentos relacionados à contração muscular, ainda tem sido a eletromiografia.

\subsection{Sinal Mioelétrico}

O sinal EMG é formado pelo agrupamento de sinais provenientes dos músculos em atividade, sendo estes músculos os responsáveis por determinado movimento. A compreensão da sua origem, suas características e como o sinal EMG varia em função da participação de cada músculo, é fundamental para escolha e construção da técnica de captura. Sendo assim, abordaremos mais detalhes sobre estes pontos.

\subsubsection{Origem e características do sinal mioelétrico}

A origem do sinal mioelétrico encontra-se na estrutura formada por um neurônio motor (motoneurônio) e um conjunto de células musculares (fibras musculares) inervadas por ele. A Figura 4.2 ilustra um exemplo de unidade motora (UM). O sinal é gerado a partir 
da passagem de íons entre os lados interno e externo das células. Essa onda se propaga pelo tecido e recebe o nome de potencial de ação da unidade motora (MUAP).

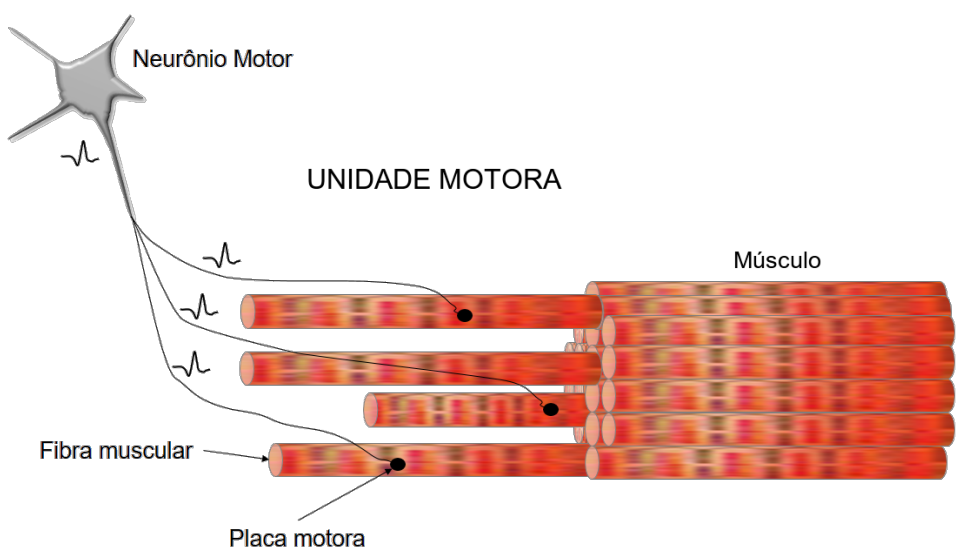

Figure 4.2. Ilustração de uma unidade motora. Fonte: próprio autor

Cada músculo envolvido no movimento dos membros, é responsável por um sinal composto pela somatória de centenas de MUAPs. Considerando que a mobilização de um determinado membro exige a participação de vários músculos, o sinal capturado na superfície apresenta-se bastante complexo. Portanto, podemos afirmar que o sinal EMG é o somatório de milhares de MUAPs. A Figura 4.3 ilustra a captura dos sinais utilizando um amplificador diferencial.

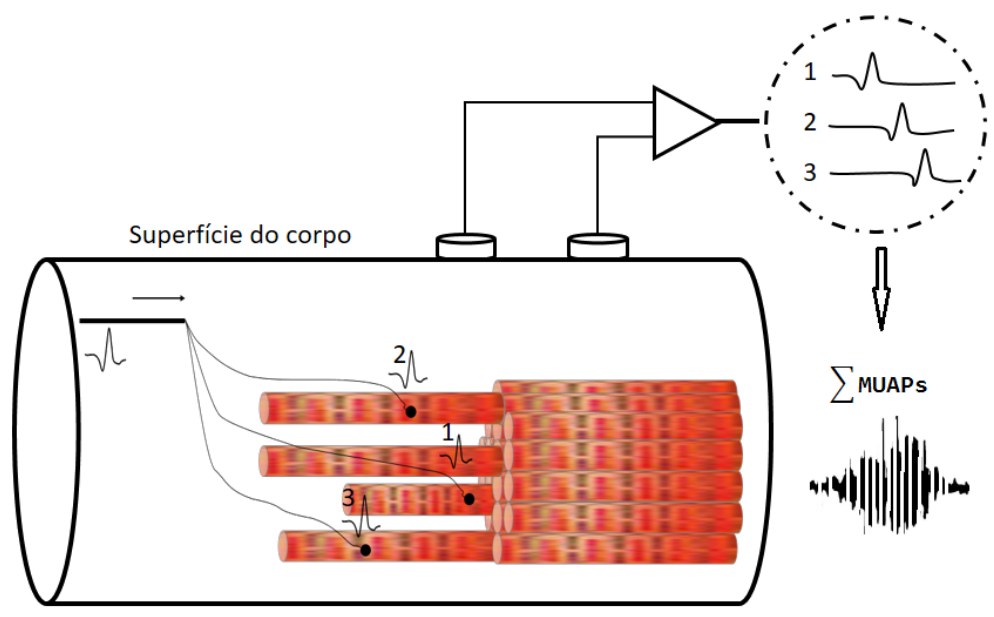

Figure 4.3. Ilustração da captura do sinal EMG formado pelos vários MUAPs. Fonte: próprio autor

A aparência do sinal capturado é semelhante ao que observamos na Figura 4.4 (A). Trata-se de um sinal praticamente aleatório, mas durante a captura em uma fase de contração e relaxamento do músculo, ele apresenta um padrão Gaussiano. Em (B), está apresentado o espectro de frequência do sinal. Embora varie de $1 \mathrm{hz}$ a $500 \mathrm{hz}$, sua faixa mais importante encontra-se entre $6 \mathrm{hz}$ e $20 \mathrm{hz}$, sendo esta a faixa de frequência onde a maioria das fibras musculares está atuando. A intensidade do sinal pode chegar aos 10 milivolts na superfície da pele quando medido em atletas (MERLETTI; PARKER, 2004). 


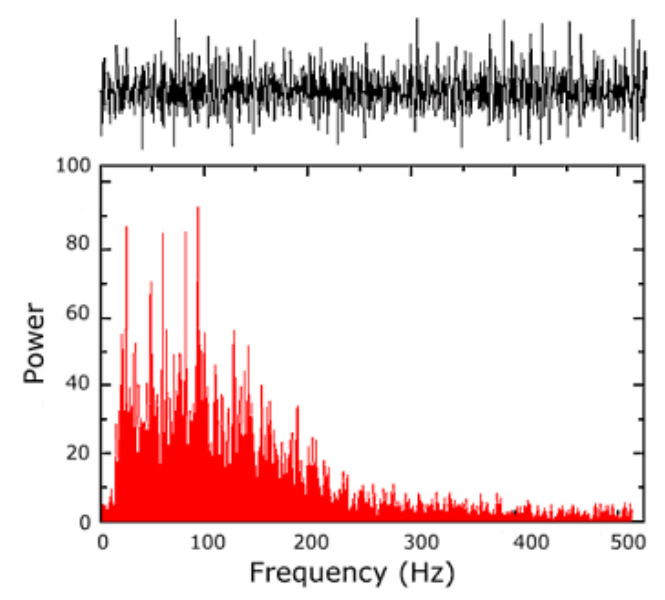

Figure 4.4. (A) sinal EMG bruto; (B) espectro de frequência do sinal. Fonte: (DE LUCA, 2002)

\subsubsection{Comportamento dos sinais na presença de movimentos}

O padrão do sinal EMG pode refletir um determinado movimento executado por um membro através de um padrão, no entanto, vários fatores podem alterar esse padrão. Por tanto, vamos abordar os fatores que consideramos mais importantes de serem controlados para que sejam aumentadas as chances de acertar quais os movimentos estão sendo executados a partir da identificação do padrão dos sinais EMG.

Eletrodos: O projeto SENIAM ${ }^{1}$ faz algumas recomendações em relação aos eletrodos para que a aquisição seja feita de forma adequada (STEGEMAN; HERMENS, 2007):

- Tamanho dos eletrodos: recomenda-se que sejam utilizados tamanhos que variam de $1 \mathrm{~mm}^{2}$ a alguns $\mathrm{cm}^{2}$. Os eletrodos maiores, principalmente seguindo o sentido das fibras musculares apresentam maior ganho e apresentam comportamento de filtro passa-baixa;

- Distância entre os eletrodos: segundo o projeto, a distância mais adequada é de $20 \mathrm{~mm}$ entre os eletrodos, com a observação de que em músculos pequenos, essa distância deve estar limitada a $\frac{1}{4}$ do tamanho do músculo.

- Posicionamento dos eletrodos: o posicionamento indicado é na região da pele sobre os ventres musculares dos principais músculos que estão participando do movimento.

- Materiais dos eletrodos: como já foi mencionado, é recomendo os eletrodos com terminais com $\mathrm{AgCl}, \mathrm{Ag}$ ou $\mathrm{Au}$.

Local da coleta: O local é algo que depende do número de eletrodos a serem utilizados e, embora sejam recomendadas a aplicação sobre os ventres musculares (regiões

\footnotetext{
${ }^{1}$ Projeto que padronizar a forma de aquisição do sinal mioelétrico de superfície (www.seniam.org)
} 
de maior volume do músculo), a escolha de quais deles serão utilizados é algo que depende de testes. O mais importante é saber que uma vez determinado o local da coleta, não poderá haver variações na colocação, visto que isto implica na alteração do padrão do sinal.

Padronização do movimento: Outro fator importante é a padronização do movimento, ou seja, o movimento precisa ser executado sempre com a mesma força, amplitude e angulações para que o padrão seja estável. Nestas condições é possível identificar o movimento a partir dos sinais capturados.

Treinamento: Chamamos de treinamento, a repetição de determinado movimento com o objetivo de conseguir um padrão de sinal que possa ser associado exclusivamente ele.

\subsection{Tratamento dos sinais mioelétricos}

Uma das condutas mais importantes quando se procura utilizar os sinais EMG para uso em próteses é o tratamento destes sinais. As várias interferências a que estão submetidos dificultam sua classificação. Assim, apresentaremos alguns dos fatores que alteram o padrão do sinal EMG e que devem ser controlados.

\subsubsection{Artefatos e ruídos presentes na aquisição dos sinais}

Os artefatos e ruídos são interferências que produzem distorções nos sinais biológicos, e que não são de interesse para um determinado estudo (FERREIRA, 2007). Condutas importantes para diminuir estas interferências são: manter os eletrodos bem fixados à pele, e os cabos bem fixados aos eletrodos para evitar movimentos; evitar exposições a campos eletromagnéticos intensos; utilizar amplificadores com alta relação sinal/ruído.

Os amplificadores são muito importantes nesse processo, visto que seus ganhos são muito altos. Valores em torno de 10 mil vezes são utilizados, visto que os sinais são da ordem de 0,3 a $10 \mathrm{mV}$, por isso a relação sinal/ruído é um fator muito importante.

\subsubsection{Alguns filtros utilizados na melhoria da qualidade dos sinais}

A filtragem dos sinais está relacionada ao uso destes sinais. Quando o objetivo é diagnóstico, a filtragem é bastante complexa e vários filtros são aplicados tanto, utilizando componentes físicos quanto utilizando filtros digitais. Nestes casos, a filtragem deve procurar eliminar inclusive os chamados cross-talk, que são os sinais oriundos dos músculos próximos ao que está sendo avaliado.

No caso do uso para próteses, a filtragem é menos exigente podendo o cross-talk, por exemplo, fazer parte do padrão do sinal EMG. Isto vai depender ainda se o sinal EMG será utilizado apenas para classificar o movimento que deu origem ao sinal, ou se será usado para medir a força e os movimentos finos. Os filtros mais utilizados no primeiro caso são o passa-baixa para evitar a produção de aliasing, e o filtro de $60 \mathrm{~Hz}$.

\subsubsection{Parte do sinal a ser considerada, dependendo do objetivo}

Durante o processo de aquisição, dependendo do objetivo (classificação do movimento ou identificação de início meio e fim do movimento) parte da aquisição pode ser descartada. 
No segundo caso, deve ser incrementado algoritmo de leitura do espectro de frequência para medir a força muscular e outros que permitissem identificar o início e o fim do movimento. Quando o uso é classificador, a parte inicial e final do sinal é desconsiderada.

\subsection{Técnicas de Identificação dos Movimentos}

É muito importante, antes de procurar identificar a técnica utilizada para identificação do movimento associado ao padrão do sinal EMG, determinar qual o objetivo da coleta. Algumas próteses com baixas funcionalidades necessitam apenas dos estados dos membros (flexão, extensão, abdução, adução etc.). Diante dessa informação, as próteses posicionam-se mimetizando o estado identificado.

Outras próteses com altas funcionalidades necessitam de informações mais detalhadas, como o momento em que o movimento foi iniciado e finalizado, assim como a variação de força durante o processo de contração e relaxamento. Para estas identificações é necessário medir não só a amplitude do sinal, como também o espectro de frequência do sinal EMG e analisar quais as frequências mais importantes em tempo de execução. Aqui, podem ser utilizadas algumas técnicas complementares, sejam elas no domínio do tempo, no domínio da frequência, ou no domínio tempo-frequencia. Podemos citar a Transformada Rápida de Fourier (FFT); Transformada Wavelet (WT) e Transformada Wavelet Packet (WPT).

Neste capítulo iremos abordar de forma resumida as técnicas para próteses simples, portanto, iremos falar das técnicas de classificação dos movimentos. Considerando que existem varias técnicas com este objetivo, citaremos apenas três delas.

\subsubsection{Redes Neurais}

Quando se escolhe as Redes neurais para fazer classificação de movimento através dos sinais EMG, não há necessidade de hardware robusto se estivermos falando de apenas dois movimentos, exemplo flexão e extensão. Podemos ver isto na construção da Mão de São Carlos (CUNHA, 2002). Já para a prótese mimetizar vários movimentos, se faz necessário o uso de Deep Learning e isto exige muito mais esforço de processamento.

A unidade funcional que deu origem ao nome da Rede Neural é o neurônio artificial (HAYKIN, 2001). A Figura 4.5 apresenta um modelo deste neurônio onde $X_{1}$ a $X_{n}$ correspondem aos sinais de entrada; $W$ representam um determinado peso que correspondem às sinapses dos neurônios biológicos; $k_{n}$ é cada um dos neurônios utilizados.

Os sinais provenientes dos neurônios são aplicados em um somador $\left(\sum\right)$ que soma todas as multiplicações $\mathrm{W}_{\mathrm{kn}}$ resultantes e direciona o resultado $U_{k}$ para uma função de ativação $(\varphi)$ que determina a saída $Y_{k}$. No modelo apresentado existe um threshold que regula esse limiar de ativação.

Matematicamente, o sinal de saída pode ser expresso conforme as equações 1 e 2. Sendo $U_{k}$ o valor de saída do somador e $Y_{k}$ a saída da função de ativação do neurônio.

As Redes Neurais podem ser aplicadas em várias áreas, entre elas podemos citar o diagnóstico de doenças musculares, a Interação Humano Computador (IHC) e as próteses. Nesta última, pode atuar na classificação de sinais EMG (AHSAN; IBRAHIMY; KHALIFA, 2012). 


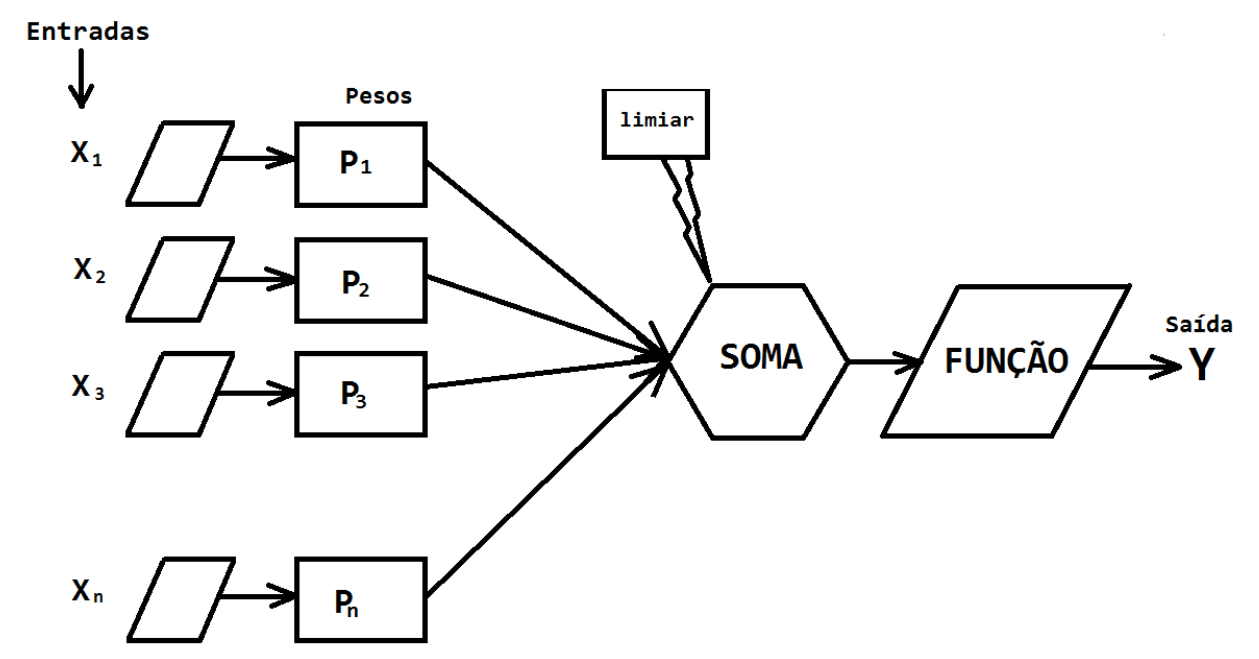

Figure 4.5. Modelo de um neurônio artificial não linear utilizado em Redes Neurais. Fonte: Modificado de (HAYKIN, 2001, p. 36)

\subsubsection{Sistema de Inferência fuzzy - FIS}

Desenvolvido por Zadeh (1965), é um método capaz de imitar a tomada decisão humana de forma mais semelhante, do que outros classificadores. A natureza não estacionária do sinal EMG tem sido um dos fatores que dificultam sua classificação, além de não ter um padrão de repetição. Isto dificulta para os classificadores estatísticos, mas o método fussy apresenta-se adaptável no reconhecimento do padrão (KHEZRI; JAHED, 2011).

Este método tem sido usado como classificador dos sinais EMG associado a outros métodos, como o feedback visual. Um projeto que fez uso da inferência fussy para controle de prótese multifuncional foi o de Ajiboye e Weir (2005).

\subsubsection{Random Forest}

O Random Forest é um método de aprendizagem supervisionado. Seu funcionamento assemelha-se a um fluxograma contendo nós com ramificações. Em cada nó é feito um teste para tomada de decisão, e isso tem um formato de árvores. A construção das árvores é mais rápida do que outros métodos utilizados para construção dos classificadores, sendo também tão preciso, ou mais, do que estes outros (SHARMA; KUMAR, 2016).

Vários algoritmos de Random Forest estão disponíveis. Citamos aqui seis deles: CHID, CART, ID3, C4.5, C5.0, Sec5 e Hunt's Algorithm. Quatro destes podem ser visto com mais detalhe na tabela 4.1. O termo Pruning faz referência à técnica de aprendizagem de máquina que otimiza o tamanho das árvores de decisão, atuando na remoção das seções que fornecem pouco poder de classificação. Já o termo Boosting faz referência ao algoritmo capaz de fazer a conversão de aprendizagem fraca em forte (PATEL; RANA, 2014).

Por fim, o Random Forest é um conjunto de árvores de decisão aleatória. Neste método cada árvore indica uma previsão da classificação, aquela que for mais indicada na floresta é eleita a previsão do método. 
Table 4.1. Comparação entre algoritmos de Árvore de Desisão.

\begin{tabular}{l|c|c|c|c}
\hline Parâmetros & ID3 & C4.5 & C5.0 & CART \\
\hline Tipo de dado & Categoria & Contínuo e Categoria & Contínuo, Categoria, Data, Tempo & Contínuo, Atributos, Data \\
Velocidade & Baixa & Maior que ID3 & Média \\
Pruning & Não & Pré-Pruning & Prét-Pruning & Post-Pruning \\
Boosting & Não suportado & Não suportado & Não suportado & Nãoortado \\
Ausência de Valores & Não lidar & Não lidar & Pode lidar & Pode lidar \\
Fórmula & Usa entropia e ganho de informação & Informação dividida e taxa de ganho & Mesmo C4.5 & índice de diversidade Gini \\
\hline
\end{tabular}

\subsection{Instruções para o processo de aquisição}

As instruções para uma aquisição adequada não se limita a condutas. Trata-se de um processo personalizado devido às variações anatômicas e fisiológicas entre os indivíduos. Assim, entendemos que se inicia na seleção ou construção própria de um equipamento de aquisição, na construção de uma interface de treinamento e das recomendações apresentadas no projeto SENIAM.

\subsubsection{Equipamento de aquisição}

A escolha ou construção do equipamento adequado depende de vários fatores como: quantos canais serão utilizados, e isto depende da quantidade de movimentos que se pretende identificar, além da quantidade de músculos envolvidos; custos, software embutido e dos filtros necessários. Equipamentos comerciais de eletromiografia geralmente não é uma boa opção pelo custo, tamanho e por apresentarem recursos dispensáveis aos projetos de próteses.

Apresentaremos aqui um exemplo de construção de um equipamento de aquisição construído pelo programa de pós-graduação em modelagem computacional e tecnologia industrial do SENAI CIMATEC em parceria com a UNCISAL e apoio da FAPESB (PEIXOTO, 2021)

Trata-se de um equipamento em fase de protótipo que contém cinco canais de aquisição. Segundo o autor, com apenas três canais é possível identificar pelo menos cinco movimentos da mão e punho. Para sua confecção foram utilizadas cinco placas comerciais de aquisição de sinal EMG modelo Myo WareTM Muscle Sensor (AT-04-001). Cada placa tem 1 canal de entrada e foram arranjada em paralelo para a composição dos 5 canais.

Além das placas de aquisição, foi utilizada uma placa Arduíno ${ }^{\circledR}$ para realizar a conversão A/D (analógico digital). Também compõem o equipamento: uma bateria interna, um regulador de tensão e os cabos com os eletrodos. O protótipo pode ser visto na Figura 4.6 cuja composição apresenta-se dividida em três partes.

\subsubsection{Algoritmo classificador}

$\mathrm{O}$ algoritmo utilizado neste projeto foi o Random Forest. O objetivo foi apenas de identificar as posições em que a mão e punho se encontravam e classificá-los conforme um grupo de posições pré-estabelecidas.

A utilização do Random Forest proporcionou um acerto de cinco posicionamentos da mão e punho que variou de $72 \%$ a $99 \%$ utilizando 3 canais. Um trabalho realizado na China utilizando a braçadeira Myo armband 8 canais para identificação de 10 movimentos da mão teve um percentual de acerto entre $73,7 \%$ a $100 \%$. 


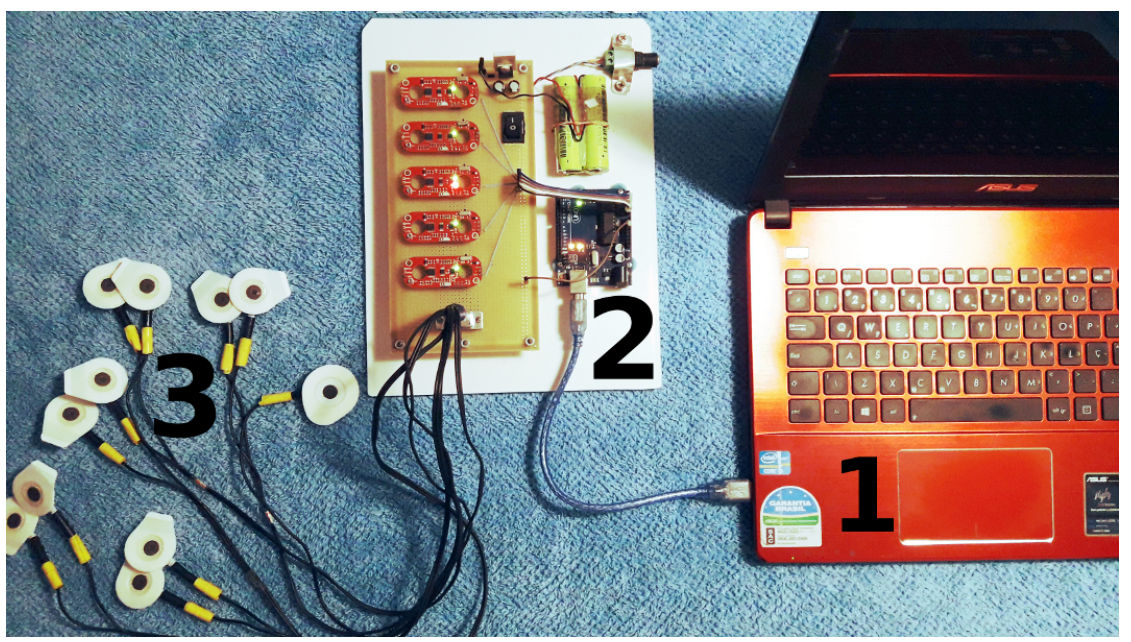

Figure 4.6. Equipamento de aquisição de sinais EMG. (1) computador, (2) placa de aquisição, (3) conjunto de canais. Fonte: (PEIXOTO, 2021)

\subsubsection{Procedimentos recomendados}

As recomendações apresentadas aqui estão em conformidade com o projeto SENIAM e em maior parte obtidas do trabalho de Peixoto (2021). A lista a seguir apresenta estas recomendações divididas em quatro partes.

\section{Cuidados gerais}

- Limpeza da pele com água e sabão para retirada do suor e produtos químicos

- Retirada de pelos

- Evitar proximidade com aparelhos que emitem campos eletromagnéticos intensos

\section{Características do sistema de aquisição}

- Utilizar eletrodos descartáveis que tenham contato de $\mathrm{Ag} / \mathrm{AgCl}$ e gel sólido, e não reutilizar estes eletrodos

- Em caso de uso prolongado do eletrodo, substituir o descartável pelo fixo

- Utilizar bateria nos equipamentos de aquisição em vez de ligar direto à rede elétrica

\section{Posicionamento dos eletrodos}

- Manter a distância de 2 a 2,5cm entre os eletrodos de cada canal

- Prender os eletrodos de forma que não fiquem instáveis

- As recomendações para a localização dos eletrodos é que sejam colocados sobre os principais ventres musculares relacionados aos movimentos realizados. 


\section{Aquisição do sinal}

- Antes das aquisições para treinamento dos algoritmos, deve-se realizar treinos com feedback para padronização dos sinais

- Não há um número ideal de aquisições previamente determinado, deve-se testar qual o número mais adequado para a aprendizagem do algoritmo. O número de aquisições e o treinamento são dois componentes relacionados e seus aumentos tendem a aumentar a eficiência do algoritmo

- Em caso do sinal ser utilizado para outros fins que não a prótese mioelétrica, escolher preferencialmente o membro predominante, de maior uso da pessoa

\section{References}

AHSAN, M. R.; IBRAHIMY, M. I.; KHALIFA, O. O. The Use of Artificial Neural Network in the Classification of EMG Signals. In: 2012 Third FTRA International Conference on Mobile, Ubiquitous, and Intelligent Computing. IEEE, 2012. p. 225-229. ISBN 978-1-4673-1956-0. Disponível em: <http: //ieeexplore.ieee.org/document/6305853/>.

AJIBOYE, A.; WEIR, R. A Heuristic Fuzzy Logic Approach to EMG Pattern Recognition for Multifunctional Prosthesis Control. IEEE Transactions on Neural Systems and Rehabilitation Engineering, v. 13, n. 3, p. 280-291, sep 2005. ISSN 1534-4320. Disponível em: <http://ieeexplore.ieee.org/document/1506815/>.

CHIANURA, A.; GIARDINI, M. E. An electrooptical muscle contraction sensor. Medical \& Biological Engineering \& Computing, v. 48, n. 7, p. 731-734, jul 2010. ISSN 0140-0118. Disponível em: <http://link.springer.com/10.1007/s11517-010-0626-x>.

CUNHA, F. L. da. Mão de São Carlos, uma prótese multifunção para membros superiores: um estudo dos mecanismos, atuadores e sensores. Tese (Doutorado) — Universidade de São Paulo, São Carlos, apr 2002. Disponível em: <http: //www.teses.usp.br/teses/disponiveis/18/18133/tde-13032006-124951/>.

DE LUCA, C. J. Surface Electromyography: detection and recording. DELSYS Incorporated, 2002. 1-10 p. Disponível em: <https://www.delsys.com/downloads/ TUTORIAL/semg-detection-and-recording.pdf $>$.

FERREIRA, É. L. C. Análise da interferência de ruídos e artefatos no processo de aquisiçao e processamento digital de um sinal biológico. Tese (Doutorado) - Univap, 2007.

HARGROVE, L.; ENGLEHART, K.; HUDGINS, B. A Comparison of Surface and Intramuscular Myoelectric Signal Classification. IEEE Transactions on Biomedical Engineering, v. 54, n. 5, p. 847-853, may 2007. ISSN 0018-9294. Disponível em: $<$ http://ieeexplore.ieee.org/document/4154997/>. 
HAYKIN, S. Redes neurais: princípios e prática. 2. ed. Porto Alegre: Bookman, 2001. 902 p. ISBN 978-85-7307-718-6.

KARMEN, G.; GABRIEL, D. A. Essentials of Electromyography. United States: Human Kinetics, 2010. 256 p. ISBN 0-7360-8550-5.

KEIDEL, M.; KEIDEL, W.-D. The Computer-Vibromyography as a Biometric Progress in Studying Muscle Function - Die Computer-Vibromyographie - Ein biometrischer Ansatz zur Messung mechanischer Muskelaktivität. Biomedizinische Technik/Biomedical Engineering, v. 34, n. 5, p. 107-116, 1989. ISSN 0013-5585. Disponível em: $<$ https://www.degruyter.com/document/doi/10.1515/bmte.1989.34.5.107/html>.

KHEZRI, M.; JAHED, M. A NeuroFuzzy Inference System for sEMG-Based Identification of Hand Motion Commands. IEEE Transactions on Industrial Electronics, v. 58, n. 5, p. 1952-1960, may 2011. ISSN 0278-0046. Disponível em: $<$ http://ieeexplore.ieee.org/document/5491165/>.

KRUEGER, E.; SCHEEREN, E. M.; NOGUEIRA-NETO, G. N.; BUTTON, V. L. d. S. N.; NOHAMA, P. Advances and perspectives of mechanomyography. Revista Brasileira de Engenharia Biomédica, v. 30, n. 4, p. 384-401, dec 2014. ISSN 1517-3151. Disponível em: <http://www.scielo.br/scielo.php?script=sci $\left\{\backslash \_\right\} \operatorname{arttext}\{\backslash \&\}$ pid= S1517-31512014000400009\{\\&\}lng=e>.

LIPPERT, L. S. Cinesiologia Clínica e Anatomia. 5. ed. Rio de Janeiro: Gua, 2013. 348 p. ISBN 978-85-277-2190-5.

MERLETTI, R.; PARKER, P. Electromyography Physiology, Engineering, and Non-Invasive Applications. New Jersey: John Wiley \& Sons, Inc., Hoboken, 2004. 493 p. ISBN ISBN 0-471-67580-6.

ORIZIO, C.; PERINI, R.; VEICSTEINAS, A. Muscular sound and force relationship during isometric contraction in man. European Journal of Applied Physiology and Occupational Physiology, v. 58, n. 5, p. 528-533, mar 1989. ISSN 0301-5548. Disponível em: <http://link.springer.com/10.1007/BF02330708>.

PATEL, B. R.; RANA, K. K. A Survey on Decision Tree Algorithm For Classification. IJEDR, v. 2, n. 1, p. 2321-9939, 2014. Disponível em: <https://www.ijedr.org/papers/ IJEDR1401001.pdf>.

PEIXOTO, A. M. Aquisição de sinais EMG da região do antebraço para uso em róteses mioelétricas: contexto, experimentos e definição de um protocolo. 105 p. Tese (Doutorado) - SENAI CIMATEC, 2021.

POZZO, M. Electromyography (EMG), Electrodes and Equipment for. In: Wiley Encyclopedia of Biomedical Engineering. Hoboken, NJ, USA: John Wiley \& Sons, Inc., 2006. Disponível em: <http://doi.wiley.com/10.1002/9780471740360.ebs1425>.

SHARMA, H.; KUMAR, S. A Survey on Decision Tree Algorithms of Classification in Data Mining. International Journal of Science and Research (IJSR), v. 5, 2016. Disponível em: <https://www.researchgate.net/publication/324941161>. 
STEGEMAN, D. F.; HERMENS, H. J. Standards for surface electromyography: the european project surface emg for non-invasive assessment of muscles (seniam). p. 108-112, 2007.

TAMEE, K.; CHAIWONG, K.; YOTHAPAKDEE, K.; YUPAPIN, P. P. Muscle sensor model using small scale optical device for pattern recognitions.(Research Article). The Scientific World Journal, v. 13, 2013. ISSN 1537-744X.

WATAKABE, M.; MITA, K.; AKATAKI, K.; ITOH, Y. Mechanical behaviour of condenser microphone in mechanomyography. Medical \& Biological Engineering \& Computing, v. 39, n. 2, p. 195-201, mar 2001. ISSN 0140-0118. Disponível em: $<$ http://link.springer.com/10.1007/BF02344804>.

YOTHAPAKDEE, K.; P.YUPAPIN, P.; TAMEE, K. Facial Gesture Measurement Using Optical Muscle Sensing System. Nano Biomedicine and Engineering, v. 7, n. 4, jan 2016. ISSN 2150-5578. Disponível em: <http://nanobe.org/Data/View/268?type=100>.

ZADEH, L. Fuzzy sets. Information and Control, v. 8, n. 3, p. 338-353, jun 1965. ISSN 00199958. Disponível em: <https://linkinghub.elsevier.com/retrieve/pii/ S001999586590241X>. 